\title{
THE $v_{1}$-PERIODIC HOMOTOPY GROUPS OF AN UNSTABLE SPHERE AT ODD PRIMES
}

\author{
ROBERT D. THOMPSON
}

\begin{abstract}
The mod $p \quad v_{1}$-periodic homotopy groups of a space $X$ are defined by considering the homotopy classes of maps of a Moore space into $X$ and then inverting the Adams self map. In this paper we compute the mod $p v_{1}$ periodic homotopy groups of an odd dimensional sphere, localized at an odd prime. This is done by showing that these groups are isomorphic to the stable $\bmod p v_{1}$-periodic homotopy groups of $B \Sigma_{p}^{2(p-1) n}$, the $2(p-1) n$ skeleton of the classifying space for the symmetric group $\Sigma_{p}$. There is a map $\Omega^{2 n+1} S^{2 n+1} \rightarrow \Omega^{\infty}\left(J \wedge B \Sigma_{p}^{2(p-1) n}\right)$, where $J$ is a spectrum constructed from connective $K$-theory, and the image in homotopy is studied.
\end{abstract}

\section{INTRODUCTION}

The main objective of this paper is to compute the $v_{1}$-periodic homotopy groups of an unstable sphere at odd primes. This is done by carrying out an odd primary analogue of a program developed for $p=2$ by Mark Mahowald [M1]. As is often the case, the calculations done here at odd primes are simpler than their $p=2$ counterparts, so another purpose of this work could be to illuminate the $p=2$ case.

For a 2-connected space $X$ the $v_{1}$-periodic homotopy groups of $X$ are defined as follows. Let $M^{n}$ denote a mod $p$ Moore space with top cell in dimension $n$, where $p$ is an odd prime. Let $\pi_{n}(X ; \mathbf{Z} / p)$ denote the mod $p$ homotopy groups of $X$, i.e., the homotopy classes of maps from $M^{n}$ to $X$. These homotopy groups are $\mathrm{Z} / p$ vector spaces. In his seminal paper $J(X)$ IV, [A], J. F. Adams constructed a map $v_{1}: M^{2(p-1)+n} \rightarrow M^{n}, n \geq 4$, which induces an isomorphism in $K$-theory. In fact, it is shown in [CN] that $v_{1}$ can be constructed for $n=3$. We can define a $\mathbf{Z} / p\left[v_{1}\right]$-module structure on $\pi_{*}(X ; \mathbf{Z} / p)$ by setting $v_{1} \alpha=\alpha \circ v_{1}$ for each $\alpha: M^{n} \rightarrow X$. Then the mod $p v_{1}$-periodic homotopy groups of $X$ are defined to be

$$
\pi_{*}(X ; \mathbf{Z} / p) \otimes_{\mathbf{Z} / p\left[v_{1}\right]} \mathbf{Z} / p\left[v_{1}, v_{1}^{-1}\right],
$$

which we denote simply by $v_{1}^{-1} \pi_{*}(X ; \mathbf{Z} / p)$. Inverting $v_{1}$ annihilates the subgroup of $v_{1}$-torsion elements and the resulting periodic groups are in general much smaller and hopefully tractable.

Received by the editors April 11, 1988.

1980 Mathematics Subject Classification (1985 Revision). Primary 55R35.

The author was partially supported by the NSF. 
To compute $v_{1}^{-1} \pi_{*}\left(S^{2 n+1} ; \mathbf{Z} / p\right)$, we show that it is isomorphic to the stable $v_{1}$-periodic homotopy groups of $\left(B \Sigma_{p}\right)^{2 n(p-1)}$, where $\left(B \Sigma_{p}\right)^{2 n(p-1)}$ denotes the $2 n(p-1)$ skeleton of the classifying space for the symmetric group on $p$ objects, localized at $p$. Set $q=2(p-1)$. Denoting $\Omega^{\infty} \Sigma^{\infty}(X)$ by $Q(X)$, there is a Hopf-James-Snaith map

$$
s: \Omega^{2 n+1} S^{2 n+1} \rightarrow Q\left(\left(B \Sigma_{p}\right)^{q n}\right)
$$

and the main theorem of this paper is

Theorem 1.1.

$$
s_{*}: v_{1}^{-1} \pi_{*}\left(\Omega^{2 n+1} S^{2 n+1} ; \mathbf{Z} / p\right) \rightarrow v_{1}^{-1} \pi_{*}\left(Q\left(\left(B \Sigma_{p}\right)^{q n}\right) ; \mathbf{Z} / p\right)
$$

is an isomorphism for all $n \geq 1$.

This is proved by induction on the dimension of the sphere, after first computing the $v_{1}$-periodic homotopy groups of the fiber of the double suspension map. More precisely, let $W(n)$ denote the fiber of the map $S^{2 n-1} \rightarrow \Omega^{2} S^{2 n+1}$. In [C], Fred Cohen constructs a map $W(n) \rightarrow Q\left(M^{2 n p-2}\right)$. In $\S 2$, following Mahowald [M1, M2, MT], we show that this map induces an isomorphism of $v_{1}$-periodic homotopy groups. This is proved by constructing a tower of fibrations for $W(n)$ which yields a spectral sequence having as its $\mathrm{E}_{2}$-term the homology of a certain subquotient of the lambda algebra. This $E_{2}$-term was analyzed in detail in [M2] for $p=2$ and in [HM1] for $p$ odd and was shown to be isomorphic to the stable $\mathrm{E}_{2}$-term for the Moore space after inverting $v_{1}$. We show that Cohen's map induces this isomorphism, yielding the result in homotopy. The significance of this is that the unstable $v_{1}$-periodic homotopy groups of the space $Q(M)$ are the stable $v_{1}$-periodic homotopy groups of $M$, and these stable groups have been calculated by H. Miller in [Mil].

The stable $v_{1}$-periodic homotopy groups of a spectrum are described very conveniently in terms of $J$-homology. The spectrum $J$ is a certain spectrum constructed from connective $K$-theory such that the homotopy groups of $J$ at an odd prime are isomorphic to the image of the classical $J$-homomorphism [W, A]. In $\S 3$ the basic properties of the $J$ spectrum and the computation of $J_{*}\left(B \Sigma_{p}^{m}\right)$ are reviewed and the following explicit corollary is derived:

Corollary 1.2. $v_{1}^{-1} \pi_{*}\left(\Omega^{2 n+1} S^{2 n+1} ; \mathbf{Z} / p\right)$ is isomorphic to a free module over $\mathbf{Z} / p\left[v_{1}, v_{1}^{-1}\right]$ on four generators in dimensions $q n-2, q n-1, q n-1, q n$.

So far we have only considered homotopy with $\mathrm{Z} / p$ coefficients. We would like to describe the homotopy classes in $\pi_{*}\left(S^{2 n+1}\right)$ with integral coefficients (localized at $p$ ) which are detected by $J_{*}\left(\left(B \Sigma_{p}\right)^{q n}\right)$. The groups $J_{*}\left(B \Sigma_{p}^{q n}\right)$ are cyclic and concentrated in dimensions of the form $q k-1$ and $q k-2$. The following is proved in $\S 4$. 
Theorem 1.3. The composite

is surjective for $k \geq 1$. The composite

$$
\pi_{q k-1}\left(\Omega^{2 n+1} S^{2 n+1}\right) \rightarrow \pi_{q k-1}^{S}\left(B^{q n}\right) \rightarrow J_{q k-1}\left(B^{q n}\right)
$$

$$
\pi_{q k-2}\left(\Omega^{2 k+1} S^{2 n+1}\right) \rightarrow \pi_{q k-2}^{S}\left(B^{q n}\right) \rightarrow J_{q k-2}\left(B^{q n}\right)
$$

is surjective if $k>n+\nu(k)$.

This appears as conjecture 1.5.21(a) in [R1] and there is a discussion there and in [R2] as to how it pertains to calculations with the EHP spectral sequence. Theorem 1.3 is closely related to results obtained independently by $M$. Bendersky [Be] and B. Gray [G1, G2].

This paper is distilled from the author's University of Washington Ph.D. thesis. The author wishes to thank Doug Ravenel, Mark Mahowald, Haynes Miller, and Don Davis for helpful discussions on this and other related topics. Also the author wishes to thank the referee for many helpful suggestions; in particular the referee and Mark Mahowald suggested the proof given here of 2.29 , which is an improvement over the original proof.

\section{THE PROOF OF THEOREM 1.1}

Assume that all spaces are localized at a fixed odd prime $p$. Theorem 1.1 is proved by analyzing the fiber of double suspension. More precisely, let $W(n)$ denote the fiber of the map

$$
S^{2 n-1} \rightarrow \Omega^{2} S^{2 n+1}
$$

which induces the double suspension homomorphism. We have a commutative diagram

$$
\begin{aligned}
& \Omega^{2 n-1} W(n) \longrightarrow \Omega^{2 n-1} S^{2 n-1} \longrightarrow \Omega^{2 n+1} S^{2 n+1} \\
& \downarrow w_{n} \quad \downarrow^{s_{n-1}} \quad \downarrow_{n} \\
& Q\left(M^{q n-1}\right) \longrightarrow Q\left(B \Sigma_{p}^{q(n-1)}\right) \longrightarrow Q\left(B \Sigma_{p}^{q n}\right)
\end{aligned}
$$

where the top row is $\Omega^{2 n-1}$ applied to the fiber sequence defining $W(n)$, and the bottom row is a quasifibration because the functor $Q$ converts cofibrations to quasifibrations. The map $w_{n}$ is the induced map of fibers. The commutativity of the right-hand square is proved in [Ku]. Note that $W(1)$ is homotopy equivalent to $\Omega_{0}^{3} S^{3}$. By applying $v_{1}^{-1} \pi_{*}(; \mathbf{Z} / p)$ to diagram $(2.1)$ we get a long exact sequence in $v_{1}$-periodic homotopy groups from the top row, and this maps to a long exact sequence for the bottom row. Therefore Theorem 1.1 follows immediately from induction on $n$ and the five lemma and the following theorem:

Theorem 2.2. The induced homomorphism

$$
w_{*}: v_{1}^{-1} \pi_{*}\left(\Omega^{2 n-1} W(n) ; \mathbf{Z} / p\right) \rightarrow v_{1}^{-1} \pi_{*}\left(Q\left(M^{q n-1}\right) ; \mathbf{Z} / p\right)
$$

is an isomorphism for $n \geq 1$. 
The motivation for Theorem 2.2 follows from an algebraic version of the theorem involving the lambda algebra [M2, HM1]. In order to explain this we will begin by summarizing some basic facts about the lambda algebra.

A subquotient of the lambda algebra. For an odd prime $p$ the lambda algebra $\Lambda$ is a bigraded $\mathbf{Z} / p$-algebra generated by elements $\left\{\lambda_{i}\right\}$ for $i \geq 1$ and subject to certain multiplicative relations called "Adem relations." See [6A] or [HM1] for details.

We will index elements by $(s, t)$ where $s$ is the homological degree and $t-s$ is the stem degree. Thus $\lambda_{i}$ has bidegree $(1, q i)$ and $\mu_{i}$ has bidegree $(1, q i+1)$. Using the Adem relations one can show that $\Lambda$ has a $\mathbf{Z} / p$ basis consisting of monomials in $\lambda_{i}$ and $\mu_{i}$ which are admissible; in other words which satisfy the following conditions:

(i) whenever $\lambda_{i} \lambda_{j}$ or $\lambda_{i} \mu_{j}$ occurs then $j<p i$,

(ii) whenever $\mu_{i} \lambda_{j}$ or $\mu_{i} \mu_{j}$ occurs then $j \leq p i$.

$\Lambda$ is a differential graded algebra and $\Lambda$ is filtered by subcomplexes $\Lambda(n)$, $n \geq 0$, defined by

(i) If $n=2 m, \Lambda(n)$ is the subspace spanned by admissible monomials beginning with $\lambda_{i}$ for $i \leq m$ or $\mu_{i}$ for $i<m$.

(ii) If $n=2 m+1, \Lambda(n)$ is the subspace spanned by admissible monomials beginning with $\lambda_{i}$ for $i \leq m$ or $\mu_{i}$ for $i \leq m$.

In fact $\Lambda(n)$ turns out to be a subalgebra. For a proof see [HM1]. The relevance of $\Lambda(n)$ to homotopy groups is the topic of [6A]. There it is proved that $\Lambda(n)$ is the $\mathrm{E}_{1}$-term of an unstable Adams spectral sequence for $S^{n}$. The inclusion $\Lambda(n) \rightarrow \Lambda(n+1)$ is the map of $\mathrm{E}_{1}$-terms induced by the suspension map $S^{n} \rightarrow \Omega S^{n+1}$. We will go into more detail about unstable Adams spectral sequences later, but for now we confine our attention to the algebraic picture. Define $\Lambda(W(n))$ to be the quotient chain complex

$$
\Lambda(2 n+1) / \Lambda(2 n-1) .
$$

Thus we have a short exact sequence of chain complexes

$$
0 \rightarrow \Lambda(2 n-1) \rightarrow \Lambda(2 n+1) \rightarrow \Lambda(W(n)) \rightarrow 0 .
$$

By (2.3) we have short exact sequences of chain complexes

$$
\begin{aligned}
& 0 \rightarrow \Lambda(2 n-1) \rightarrow \Lambda(2 n) \stackrel{h}{\rightarrow} \bar{\lambda}_{n} \Lambda(2 p n-1) \rightarrow 0, \\
& 0 \rightarrow \Lambda(2 n) \rightarrow \Lambda(2 n+1) \stackrel{h}{\rightarrow} \bar{\mu}_{n} \Lambda(2 p n+1) \rightarrow 0 .
\end{aligned}
$$

Here $\bar{\lambda}_{n} \Lambda(2 p n-1)$ is defined to be a 1-dimensional $\mathbf{Z} / p$ vector space with basis element $\bar{\lambda}_{n}$ in bidegree $(0,0)$ and trivial differential, tensored with $\Lambda(2 p n-1) \cdot \bar{\mu}_{n} \Lambda(2 p n+1)$ is defined similarly only we give $\bar{\mu}_{n}$ bidegree $(0,1)$. 
The maps $h$ and $\bar{h}$ are defined by

$$
\begin{aligned}
& h(\nu)= \begin{cases}0 & \text { if } \nu \neq \lambda_{n} \alpha \text { for any } \alpha, \\
\bar{\lambda}_{n} \alpha & \text { if } \nu=\lambda_{n} \alpha \text { for some } \alpha,\end{cases} \\
& \bar{h}(\nu)= \begin{cases}0 & \text { if } \nu \neq \mu_{n} \alpha \text { for any } \alpha, \\
\bar{\mu}_{n} \alpha & \text { if } \nu=\mu_{n} \alpha \text { for some } \alpha .\end{cases}
\end{aligned}
$$

It is easy to check that (2.5) and (2.6) are indeed short exact sequences of chain complexes. The reader familiar with the odd primary EHP sequence will immediately recognize (2.5) and (2.6) as algebraic analogues.

Furthermore we have the following diagram of chain complexes:

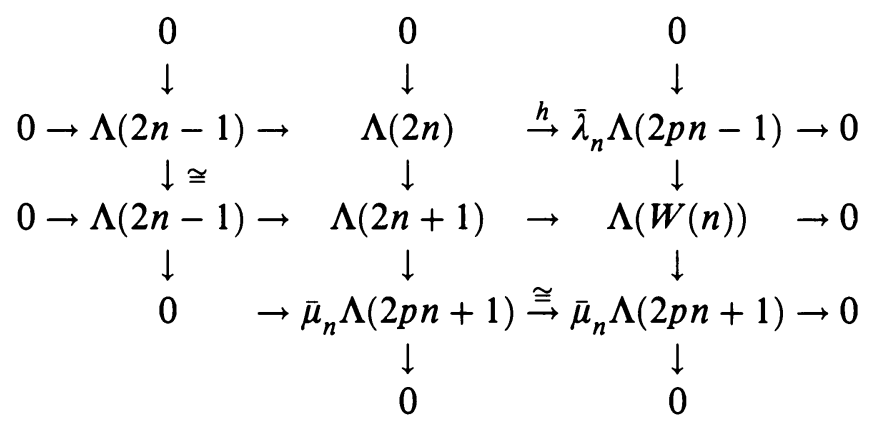

which reveals an isomorphism

$$
\Lambda(W(n)) \cong \bar{\lambda}_{n} \Lambda(2 p n-1) \oplus \bar{\mu}_{n} \Lambda(2 p n+1) .
$$

Since $\bar{\lambda}_{n}$ and $\bar{\mu}_{n}$ are $\lambda_{n}$ and $\mu_{n}$ reindexed, the above groups have been indexed so that for each $W(n)$, the lower left corner occurs at $(s, t)=(0,0)$; i.e., $W(n)^{s, t}=0$ if $s<0$ or $t-s<0$ and $W(n)^{0,0}=\mathbf{Z} / p$.

In [HM1] the differential in $\Lambda(W(n))$ is described in terms of the splitting (2.8) as follows:

Proposition 2.9 (Proposition 2.10 of [HM1]). Let $n>0, \alpha \in \Lambda(2 p n-1)$, $\nu \in \Lambda(2 p n+1)$. Then in $\Lambda(W(n))$

$$
d\left(\bar{\lambda}_{n} \alpha+\bar{\mu}_{n} \nu\right)=\bar{\lambda}_{n}\left(d \alpha+\mu_{0} \nu\right)-\bar{\mu}_{n} d \nu .
$$

From this it follows that the inclusion given by

$$
\bar{\lambda}_{n} \alpha+\bar{\mu}_{n} \nu \rightarrow \bar{\lambda}_{n+1} \alpha+\bar{\mu}_{n+1} \nu
$$

is a map of chain complexes of bidegree $(0,0)$ and induces a map in homology

$$
H^{s, t}(\Lambda(W(n))) \rightarrow H^{s, t}(\Lambda(W(n+1))) .
$$

Furthermore $\bigcup_{n} \Lambda(W(n))$ is an $\mathrm{E}_{1}$-term for the stable homotopy of the Moore spectrum $M$.

We can now state the main theorem of [HM1]. 
Theorem 2.11 (Metastability theorem of [HM1]). For $1 \leq n \leq k$ the induced map in homology

$$
H^{s, t}(\Lambda(W(n))) \rightarrow H^{s, t}(\Lambda(W(k)))
$$

is an isomorphism for

$$
t-s<2\left(p^{2}-1\right)(s-2)+p q n-2 p-2
$$

and an epimorphism for

$$
t-s \leq 2\left(p^{2}-1\right)(s-2)+p q n-2 p-2 .
$$

In the $(t-s, s)$ plane this is an isomorphism above a line of slope $1 / 2\left(p^{2}-1\right)$. A $\mathbf{Z} / p\left[v_{1}\right]$-module structure is defined on $\Lambda(W(n))$ as follows:

Definition 2.12. If $\bar{\lambda}_{n} \alpha+\bar{\mu}_{n} \nu \in \Lambda(W(n))$ define

$$
v_{1}\left(\bar{\lambda}_{n} \alpha+\bar{\mu}_{n} \nu\right)=-\bar{\mu}_{n}\left(\lambda_{1} \alpha+\mu_{1} \nu\right) \text {. }
$$

It is readily verified using the Adem relations that this defines $v_{1}: \Lambda(W(n)) \rightarrow$ $\Lambda(W(n))$ as a bidegree $(1, q+1)$ chain map which actually maps $\Lambda(W(n))$ to the image of $\Lambda(W(n-1))$. Since the map $v_{1}$ has $(1, q+1)$ for bidegree, if $x \in \Lambda(W(n)), v_{1}^{k} x$ is in the range of isomorphism for Theorem 2.11 for some $k$. So Theorem 2.11 has the following corollary:

Corollary 2.13. For $1 \leq n \leq k$ the induced map

$$
v_{1}^{-1} H^{s, t}(\Lambda(W(n))) \rightarrow v_{1}^{-1} H^{s, t}(\Lambda(W(k)))
$$

is an isomorphism.

Unstable Adams resolutions. To prove Theorem 2.2 we will use an unstable Adams spectral sequence type of approach. Unfortunately if we try to construct a spectral sequence for computing the homotopy groups of $W(n)$ using the standard machinery of $[\mathrm{BK}]$, the $\mathrm{E}_{2}$-term which would result would not resemble $H^{*, *}(\Lambda(W(n)))$. This difficulty will be avoided however by using Mahowald's construction of the "resolution of the fiber".

We begin by reviewing the general notion of a resolution of a space.

Definition 2.14. Let $X$ be space which is $p$-local and simply connected. By a resolution of $X$ we mean a diagram

such that:

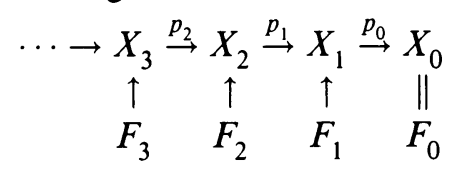

(i) Each $F_{i}$ is a generalized Eilenberg-Mac Lane space $K\left(V_{i}\right)$ where $V_{i}$ is a locally finite graded $\mathrm{Z} / p$ vector space. If $V^{j}$ denotes the homogeneous part of a graded $\mathbf{Z} / p$ vector space $V$ in degree $j$ then $K(V)=\prod_{j} K\left(V^{j}, j\right)$.

(ii) Each $F_{i} \rightarrow X_{i} \stackrel{p_{i-1}}{\rightarrow} X_{i-1}$ is a principal fibration with classifying map

$$
g_{i-1}: X_{i-1} \rightarrow B F_{i} \text {. }
$$


(iii) There are maps $f_{i}: X \rightarrow X_{i}$ and commutative diagrams

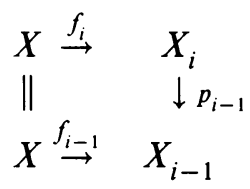

such that the induced map $f: X \rightarrow \operatorname{holim} X_{i}$ is a $p$-completion.

Definition 2.15. Given a resolution of a space $X$ there is a spectral sequence defined by

$$
\mathrm{E}_{1}^{s, t}(X)=\pi_{t-s}\left(F_{s}\right), \quad \text { where } d_{1}: \mathrm{E}_{1}^{s, t}(X) \rightarrow \mathrm{E}_{1}^{s+1, t}(X)
$$

is the homomorphism induced by the composite

$$
F_{i} \rightarrow X_{i} \rightarrow B F_{i+1} .
$$

There is a decreasing filtration of $\pi_{*} X$ defined by

$$
F_{j} \pi_{*} X=\operatorname{ker}\left(\pi_{*} X \rightarrow \pi_{*} X_{j}\right)
$$

where $\bigcap_{j \geq 0} F_{j} \pi_{*} X=0$ and the $\mathrm{E}_{\infty}$-term will be the graded vector space associated to the filtration defined above.

Of course in the generality of Definitions 2.14 and 2.15 it is difficult to say anything systematic about the $E_{2}$-term for an arbitrary space or even a family of spaces satisfying certain "nice" conditions. Furthermore such resolutions need not satisfy any "functoriality." A more strict notion would be as follows:

Definition 2.16. A resolution of a space $X$ is called an Adams resolution if, in addition to (i)-(iii) above, we require:

(iv) $\operatorname{ker} p_{i}^{*}=\operatorname{ker} f_{i}^{*}$,

(v) $f_{0}^{*}$ is surjective.

Notation. We will use the notation of [M1] and [MT]. Thus a resolution of $X$ will be denoted by $X^{\cdot}$ and the fibrations involved will be denoted by

$$
F_{i}\left(X^{*}\right) \rightarrow X_{i}\left(X^{*}\right) \rightarrow X_{i-1}\left(X^{*}\right)
$$

or simply

$$
F_{i}(X) \rightarrow X_{i}(X) \rightarrow X_{i-1}(X)
$$

if there is no ambiguity as to which resolution we are considering. Note that if $X^{*}$ is a resolution of $X$, then we can obtain a resolution of $\Omega^{k} X$ by applying $\Omega^{k}$ to $X^{\cdot}$. However, if $X^{\cdot}$ is an Adams resolution then the resolution $\Omega^{k} X^{*}$ will, in general, not be an Adams resolution.

Definition 2.17. A map of resolutions $f^{\circ}: X^{\bullet} \rightarrow Y^{*}$ covering a map $f: X \rightarrow$ $Y$ is a sequence of maps $f_{s}: X_{s}(X) \rightarrow X_{s}(Y)$ making the following diagram 
commute for $s \geq 0$ :

$$
\begin{array}{cccc}
X_{s+1}(X) & \stackrel{f_{s+1}}{\rightarrow} & X_{s+1}(Y) \\
\downarrow & & \downarrow \\
X_{s}(X) & \stackrel{f_{s}}{\rightarrow} & X_{s}(Y) \\
\uparrow & & \uparrow \\
X & \stackrel{f}{\rightarrow} & Y
\end{array}
$$

The following is proved in [M1]:

Proposition 2.19. If $f: X \rightarrow Y$ is a map and $X^{*}$ is an Adams resolution of $X$, then, for any resolution $Y^{*}$ of $Y$, there is a map of resolutions

$$
f^{\circ}: X^{\cdot} \rightarrow Y^{\circ}
$$

which covers $f$.

Thus Proposition 2.19 gives an example of the "functorial" behavior of Adams resolutions.

Remark. A map $f^{*}: X^{*} \rightarrow Y^{*}$ covering a map $f: X \rightarrow Y$ gives rise to a map of spectral sequences simply by considering the map of fibers induced by the diagram

$$
\begin{aligned}
& F_{s}(X) \rightarrow X_{s}(X) \rightarrow X_{s-1}(X) \\
& \downarrow f_{s} \quad \downarrow f_{s} \quad \downarrow f_{s-1} \\
& F_{s}(Y) \rightarrow X_{s}(Y) \rightarrow X_{s-1}(Y)
\end{aligned}
$$

$\bar{f}_{s}$ then induces a map of chain complexes:

$$
\mathrm{E}_{1}^{s, t}(X) \stackrel{\left(\vec{f}_{s}\right) *}{\rightarrow} E_{1}^{s, t}(Y) \text {. }
$$

In general it would be difficult to get a handle on the induced map of $E_{2}$ terms since these $E_{2}$-terms would not have any nice description. However in the special cases to which we will be applying these notions we will be able to determine the induced map of $E_{2}$-terms in a range of interest.

Now we give Mahowald's construction of the resolution of the fiber of a map.

Proposition 2.20. Let $f^{*}: X^{\cdot} \rightarrow Y^{\bullet}$ be a map of resolutions covering a map $f: X \rightarrow Y$ and let $(f)$ denote the homotopy theoretic fiber of $f$. Then there is a resolution of $(f)$, denoted by $(f)^{\circ}$ and a short exact sequence

$$
0 \rightarrow \mathrm{E}_{1}^{s-1, t}(Y) \rightarrow \mathrm{E}_{1}^{s, t}\left((f)^{\cdot}\right) \rightarrow \mathrm{E}_{1}^{s, t}(X) \rightarrow 0 .
$$

In the corresponding long exact sequence

$$
\cdots \rightarrow \mathrm{E}_{2}^{s, t}(f) \rightarrow \mathrm{E}_{2}^{s, t}(X) \stackrel{\partial}{\rightarrow} \mathrm{E}_{2}^{s, t}(Y) \rightarrow \mathrm{E}_{2}^{s+1, t}(f) \rightarrow \cdots
$$

the boundary map $\partial$ is the map of $\mathrm{E}_{2}$-terms induced by $f^{\circ}$.

The construction of $(f)^{\circ}$ is given in [M1]. The second statement about the boundary map $\partial$ is used implicitly in [M1] and is proved in [MT] along with a proof of the following proposition. 
Definition 2.22. A resolution $X^{*}$ of a space $X$ is called minimal if $\mathrm{E}_{2}^{s, t}\left(X^{*}\right)=$ $\mathrm{E}_{1}^{s, t}\left(X^{*}\right)$.

Proposition 2.23. For any resolution $X^{*}$, there is a minimal resolution of $\Omega X$, denoted by $\Omega X^{\circ}$, such that there are lifts of the identity map of $\Omega X$ to

$$
f^{*}: \Omega\left(X^{*}\right) \rightarrow \Omega X^{\cdot} \text { and } g^{\cdot}: \Omega X^{\cdot} \rightarrow \Omega\left(X^{*}\right)
$$

with $f^{*}$ and $g^{\cdot}$ inducing isomorphisms in $\mathrm{E}_{2}^{s, t}$.

A resolution of $W(n)$. Returning to our discussion of the homotopy of $W(n)$ we construct a resolution for $W(n)$ as follows:

Proposition 2.24. There exists a resolution of $W(n)$, denoted by $W(n)^{\circ}$, with $\mathrm{E}_{2}$-term isomorphic to $H^{s, t}(\Lambda(W(n)))$.

Proof. By the results of [6A] there is an Adams resolution of $S^{2 n-1}$ with $\mathrm{E}_{1}^{s, t}\left(S^{2 n-1}\right)$ equal to $\Lambda(2 n-1)$ as a differential vector space. Denote this by $\left(S^{2 n-1}\right)^{\circ}$. Let $\Omega^{2}\left(\left(S^{2 n+1}\right)^{\circ}\right)$ be double loops on the corresponding resolution for $S^{2 n+1}$. There is a map of resolutions $\left(S^{2 n-1}\right)^{\cdot} \rightarrow \Omega^{2}\left(S^{2 n+1}\right)^{\cdot}$ inducing the inclusion $\Lambda(2 n-1) \rightarrow \Lambda(2 n+1)$. Apply Proposition 2.20 to this map of resolutions to obtain a resolution $W(n)^{\circ}$ of $W(n)$ and compare the long exact sequence corresponding to the middle row of diagram (2.7) with the long exact sequence of Proposition 2.20:

$$
\begin{aligned}
\cdots \rightarrow H^{s, t} \Lambda(2 n-1) \stackrel{i_{i}}{\rightarrow} H^{s, t} \Lambda(2 n+1) \rightarrow H^{s, t} \Lambda( & W(n)) \\
& \rightarrow H^{s+1, t} \Lambda(2 n-1) \rightarrow \ldots
\end{aligned}
$$

and

$$
\cdots \rightarrow \mathrm{E}_{2}^{s, t}\left(S^{2 n-1}\right) \stackrel{\partial}{\rightarrow} \mathrm{E}_{2}^{s, t}\left(S^{2 n+1}\right) \rightarrow \mathrm{E}_{2}^{s, t}\left(W(n)^{\cdot}\right) \rightarrow \mathrm{E}_{2}^{s+1, t}\left(S^{2 n-1}\right) \rightarrow \cdots .
$$

Since $\partial=i_{*}$, it follows that $\mathrm{E}_{2}^{s, t}\left(W(n)^{\circ}\right) \cong H^{s, t}(\Lambda(W(n)))$ as bigraded $\mathrm{Z} / p$ vector spaces.

Now consider the following result due to Fred Cohen.

Proposition $2.25[\mathrm{C}]$. There is a map $W(n) \stackrel{c}{\rightarrow} \Omega^{2 p} W(n+1)$ which is degree one on the bottom cell. Furthermore the homotopy direct limit of the following sequence

is $Q\left(M^{2 n p-2}\right)$.

$$
W(n) \stackrel{c}{\rightarrow} \Omega^{2 p} W(n+1) \stackrel{\Omega^{2 p_{c}}}{\rightarrow} \Omega^{4 p} W(n+2) \rightarrow \cdots
$$

By applying $\Omega^{2 p}$ to the resolution $W(n+1)^{\circ}$, we obtain a resolution for $\Omega^{2 p} W(n+1)$, with $\mathrm{E}_{2}$-term $H_{*}(\Lambda(2 n+3) / \Lambda(2 n+1))$, suitably indexed.

Our goal is to produce a map of resolutions

$$
c: W(n)^{\cdot} \rightarrow \Omega^{2 p}\left(W(n+1)^{\circ}\right)
$$

which induces the isomorphism of Theorem 2.11 in a suitable range of dimensions, and use this to conclude that the map $c$ induces an isomorphism in 
$v_{1}$-periodic homotopy groups. Unfortunately, since $W(n)^{\circ}$ is not an Adams resolution we do not get $\dot{c}$ for free. However it turns out that the obstructions to obtaining $c^{\circ}$ do indeed vanish.

To see why this is true it is necessary to consider the extent to which properties (iv) and (v) of Definition 2.16 remain true after looping an Adams resolution some number of times. This is carried out at all primes in [HM2]. We will defer the discussion of that analysis as well as the proof of the following proposition until the end of the section.

Proposition 2.26. Let $W(n)^{\cdot}$ be the resolution of Proposition 2.24, reindexed so that $X_{0}=K(\mathbf{Z} / p, 2 n p-3)$. Then $W(n)^{\circ}$ has the following properties:

(i)

(ii)

$$
\begin{array}{r}
\operatorname{ker}\left\{p_{s}^{*}: H^{*} X_{s}(W(n)) \rightarrow H^{*} X_{s+1}(W(n))\right\} \\
\quad=\operatorname{ker}\left\{f_{s}^{*}: H^{*} X_{s}(W(n)) \rightarrow H^{*} W(n)\right\} .
\end{array}
$$

$$
\begin{aligned}
& f_{s}^{k}: H^{k} X_{s}(W(n)) \rightarrow H^{k} W(n) \text { is surjective for } \\
& k<(2 n p-3) p^{s+1}+(p-2) p^{s} .
\end{aligned}
$$

We now construct a map of resolutions.

Theorem 2.27. Let $\Omega^{2 p} W(n+1)^{\circ}$ denote the minimal resolution (as in Proposition 2.23) associated to $\Omega^{2 p}\left(W(n+1)^{*}\right)$. Then there exists a map of resolutions $c^{\circ}: W(n)^{\circ} \rightarrow \Omega^{2 p} W(n+1)^{\circ}$ covering the map $c: W(n) \rightarrow \Omega^{2 p} W(n+1)$.

Proof. Since $c$ is nontrivial in cohomology in the bottom dimension $2 n p-3$, there is a map $c_{0}$ making the following diagram commute:

$$
\begin{array}{ccc}
X_{0}(W(n)) & \stackrel{c_{0}}{\longrightarrow} & X_{0} \Omega^{2 p}(W(n+1)) \\
\uparrow f_{0} & & \uparrow f_{0} \\
W(n) & \stackrel{c}{\longrightarrow} & \Omega^{2 p} W(n+1)
\end{array}
$$

Now assume, inductively, that we have a map $c_{s}$ so that the outer rectangle in the following diagram commutes:

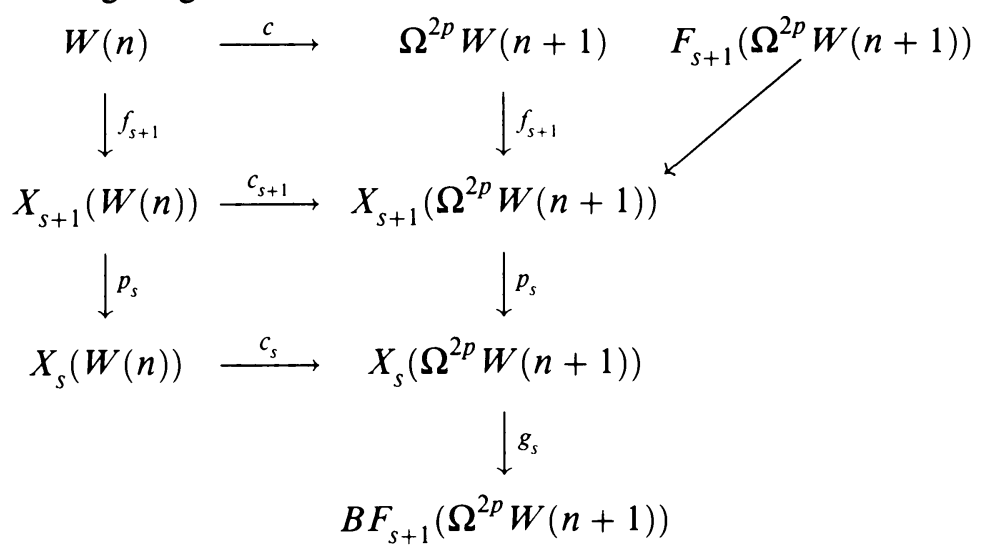


We wish a map $c_{s+1}$ making both the top half rectangle and the bottom half rectangle commute. Consider the cohomology classes in $H^{*} X_{s}(W(n))$ represented by the map $g_{s} c_{s}$. These classes are in the kernel of $f_{s}^{*}=\left(p_{s} f_{s+1}\right)^{*}$ by commutativity of the outer rectangle and the fact that $g_{s} p_{s}$ is null. Hence they are in the kernel of $p_{s}^{*}$ by Proposition 2.26(i). So there exists a map $\tilde{c}_{s+1}: X_{s+1}(W(n)) \rightarrow X_{s+1}\left(\Omega^{2 p} W(n+1)\right)$ making the lower rectangle commute. The upper rectangle need not commute. The difference $\tilde{c}_{s+1} f_{s+1}-f_{s+1} c$ lifts to a map $d: W(n) \rightarrow F_{s+1}\left(\Omega^{2 p} W(n+1)\right)$ which represents cohomology classes in $H^{*} W(n)$. Now, since $\Omega^{2 p} W(n+1)^{\bullet}$ is minimal,

$$
\pi_{*}\left(F_{s+1}\left(\Omega^{2 p} W(n+1)\right)\right) \cong H^{s+1, *}(\Lambda(W(n+1))) .
$$

By (2.8),

$$
H^{s+1, *}(\Lambda(W(n+1))) \cong H^{s+1, *}(\Lambda(2(n+1) p-1) \oplus \Lambda(2(n+1) p+1)) .
$$

So we can use this to estimate the dimension of the highest dimensional cohomology class given by $d$.

$\Lambda_{s+1}(2(n+1) p+1)$ has a basis of admissible monomials $\kappa_{i_{1}} \kappa_{i_{2}} \cdots \kappa_{i_{s+1}}$, where $\kappa_{i_{j}}=\lambda_{i_{j}}$ or $\mu_{i_{j}}, i_{1} \leq(n+1) p$, and $i_{k} \leq p i_{k-1}$. So the dimension of the largest such monomial is

$$
q(n+1)\left(p+p^{2}+\cdots+p^{s+1}\right)+2 n p-2 .
$$

But $f_{s+1}^{*}: H^{*} X_{s+1}(W(n)) \rightarrow H^{*} W(n)$ is surjective through dimensions

$$
(2 n p-3) p^{s+2}+(p-2) p^{s+1}
$$

by Proposition 2.26(ii), which is larger than the quantity in (2.28), unless $p=3$ and $n=1$. Hence $d$ extends to map $\tilde{d}: X_{s+1}(W(n)) \rightarrow F_{s+1}\left(\Omega^{2 p} W(n+1)\right)$. So if we define $c_{s+1}=\tilde{c}_{s+1}-i_{s+1} \tilde{d}$, then $c_{s+1}$ will have the properties we seek, completing the proof of Theorem 2.27 except in the special case of $p=3$ and $n=1$. To treat the case where $n=1$, note that the map $W(1) \rightarrow \Omega^{2 p} W(2)$ can be constructed to factor as $W(1) \rightarrow \Omega^{3} S^{2 p+1}\{p\} \rightarrow \Omega^{2 p} W(2)[C]$, where the middle space is the fiber of the $p$ th power map. These maps deloop, see [Se] for example, so we have $B W(1) \rightarrow \Omega^{2} S^{2 p+1}\{p\} \rightarrow \Omega^{2 p-1} W(2)$. Using Proposition 2.20 , construct resolutions for each of these spaces and a dimensional analysis as above shows that the delooped maps are covered by maps of resolutions.

Before completing the proof of Theorem 2.2 we state a lemma whose proof is given at the end of the section.

Lemma 2.29. The map of resolutions $\dot{c}$ constructed in Theorem 2.27 induces in $\mathrm{E}_{2}$-terms the map of (2.10).

To complete the proof of Theorem 2.2 let $F_{n}$ be the fiber of $c: W(n) \rightarrow$ $\Omega^{2 p} W(n+1)$. By Proposition 2.20 we have a resolution $\left(F_{n}\right)^{*}$, and by the long exact sequence of $E_{2}$-terms, the $E_{2}$-term of $\left(F_{n}\right)^{\cdot}$ has a vanishing line of slope 
$1 /\left(p^{2}-p-1\right)$. Given a mod $p$ homotopy class $\alpha: M^{k} \rightarrow F_{n}$, with filtration $s$, the filtration of $v_{1} \alpha$ must be at least $s+1$, since $v_{1}: M^{k+q} \rightarrow M^{k}$ is trivial in cohomology. For any class $\alpha \in \pi_{*}\left(F_{n} ; \mathbf{Z} / p\right), v_{1}^{j} \alpha$ has filtration above the vanishing line for some $j$, hence must be 0 . So $v_{1}^{-1} \pi_{*}\left(F_{n} ; \mathbf{Z} / p\right)=0$ and by the long exact sequence in homotopy $c$ induces an isomorphism in mod $p v_{1}$ periodic homotopy. It follows by Proposition 2.25 that there is an isomorphism

$$
v_{1}^{-1} \pi_{*}(W(n) ; \mathbf{Z} / p) \cong v_{1}^{-1} \pi_{*}^{S}\left(M_{2 n p-3} ; \mathbf{Z} / p\right) .
$$

Now observe that the map

$$
\Omega^{2 n-1} c: \Omega^{2 n-1} W(n) \rightarrow Q\left(M^{q n-1}\right)
$$

is not necessarily the same as the map

$$
w_{n}: \Omega^{2 n-1} W(n) \rightarrow Q\left(M^{q n-1}\right)
$$

of (2.1). However the $v_{1}$-periodic homotopy of the source and target are isomorphic. To finish the proof of Theorem 2.2 we must invoke the calculation of $v_{1}^{-1} \pi_{*}^{S}\left(M^{q n-1} ; \mathbf{Z} / p\right)$ given in [Mi1].

Theorem 2.31 (Miller [Mi1]). Let $K$ denote the graded field $\mathbf{Z} / p\left[v_{1}, v_{1}^{-1}\right]$. Then $v_{1}^{-1} \pi_{*}^{S}\left(M_{0} ; \mathbf{Z} / p\right)$ is a free $K$ module on four generators in dimensions $-2,-1,-1,0$.

One readily checks that the map $w_{n}$ of (2.1) maps these four generators nontrivially in mod $p$ homotopy, which gives Theorem 2.2.

Now we discuss the proof of Proposition 2.26.

Looping Adams resolutions. The topic of [HM2] is the effect of applying the functor $\Omega^{k}$ to an Adams resolution for a space $X$, where $X$ satisfies the condition that the cohomology of $X$ is a free unstable algebra over the Steenrod algebra. For a precise characterization of free unstable $\mathscr{A}$-algebras, see [MP1, Ba]. Another account of this material can be found in [H]. At an odd prime $p$, $S^{2 n+1}$ is such a space so the main theorem of [HM2] is applicable. The result is

Proposition 2.32 [HM2]. Let $X^{\cdot}$ be an Adams resolution for $S^{2 n+1}$ and assume that $k<2 n+1$.

(i) The homomorphism

$$
\left(\Omega^{k} f_{s}\right)^{i}: H^{i}\left(X_{s}\left(\Omega^{k} S^{2 n+1}\right)\right) \rightarrow H^{i}\left(\Omega^{k} S^{2 n+1}\right)
$$

is surjective if

$$
\begin{gathered}
i<(2 n+1-k) p^{s+1} \quad \text { for } k \text { odd }, \\
i<(2 n+1-k) p^{s+1}+(p-2) p^{s} \quad \text { for } k \text { even } .
\end{gathered}
$$

(ii) $\operatorname{ker}\left(\Omega^{k} f_{s}\right)^{*}=\operatorname{ker}\left(\Omega^{k} p_{s}\right)^{*}$. 
For an unstable $\mathscr{A}$ module $M$, let $\Omega M$ denote the algebraic loop functor applied to $M$, and let $U(M)$ denote the free unstable $\mathscr{A}$-algebra generated by $M$. Suppose $R$ is some unstable $\mathscr{A}$-algebra and suppose in addition that $M$ is a module over $R \odot \mathscr{A}$, the semitensor product ring. Then $U_{R}(M)$ denotes the free $R \odot \mathscr{A}$-algebra generated by $M$. See [MP1, 2] for precise definitions of these notions.

Suppose we have a fibration $F \rightarrow E \rightarrow B$ which is the pullback, by a map $g: B \rightarrow B_{0}$, of the path-loop fibration $\Omega B_{0} \rightarrow P B_{0} \rightarrow B_{0}$, where $B_{0}$ is a generalized Eilenberg-Mac Lane space and $H^{*}\left(B_{0}\right)=U(Q)$. A basic problem has been to compute $H^{*}(E)$ in terms of $H^{*}(B)$ and the map $g$. Suppose $H^{*}(B)=U(Y)$ for some unstable projective $\mathscr{A}$ module $Y$ and assume $g^{*}(Q) \subseteq Y$. Let $R=H^{*}(B) / \operatorname{ker} p^{*}$. Thus $H^{*}(E)$ is an unstable $R \odot \mathscr{A}$ algebra. In [MP1, MP2, Ba] it is proved that

(i) there is a short exact sequence of $R \odot \mathscr{A}$-modules

$$
0 \rightarrow R \rightarrow N \stackrel{\delta}{\rightarrow} M \rightarrow 0
$$

and $H^{*}(E) \cong U_{R}(N)$.

(ii) $R \cong U\left(\left.\operatorname{coker} g^{*}\right|_{Q}\right)$.

(iii) $M \cong R \otimes \Sigma \Omega\left(\left.\operatorname{ker} g^{*}\right|_{Q}\right)$.

It is convenient to modify this as follows:

Letting $Q^{\prime}=\left.\operatorname{ker} g^{*}\right|_{Q}$, there is an inclusion

$$
\Sigma \Omega Q^{\prime} \rightarrow R \otimes \Sigma \Omega Q^{\prime} \text {. }
$$

If $G$ is the submodule of $N$ given by $G=\delta^{-1}\left(\Sigma \Omega Q^{\prime}\right)$, then there is a short exact sequence of unstable $\mathscr{A}$-modules

$$
0 \rightarrow R \rightarrow G \rightarrow \Sigma \Omega Q^{\prime} \rightarrow 0 .
$$

This is called the fundamental sequence of the fibration $(E, p, F)$. Note that by tensoring with $R,(2.33)$ can be recovered from (2.34) as in $\S 15$ of [MP2].

Suppose $\left\{X_{s}\right\}$ is an Adams resolution of a space $X$, such that $H^{*}(X)=$ $U(M)$ for some unstable $\mathscr{A}$-module $M$. Let

$$
M \leftarrow P_{0} \stackrel{d_{0}}{\leftarrow} P_{1} \stackrel{d_{1}}{\leftarrow} P_{2} \leftarrow \cdots
$$

be a free resolution of $M$. Thus each $P_{s}$ is a free unstable $\mathscr{A}$-module and (2.35) is an acyclic chain complex. Suppose $\left\{X_{s}\right\}$ realizes $\left\{P_{s}\right\}$, i.e., $H^{*}\left(F_{s}\right)=$ $U\left(\Omega^{s} P_{s}\right)$ and $F_{s} \rightarrow X_{s} \stackrel{g_{s}}{\rightarrow} B F_{s+1}$ induces in cohomology the differential

$$
\Omega^{s} P_{s+1} \stackrel{\Omega^{s} d_{s}}{\rightarrow} \Omega^{s} P_{s}
$$

Now loop the resolution $\left\{X_{s}\right\} k$ times to obtain a resolution of $\Omega^{k} X$ in the sense of Definition 2.14. This will not be an Adams resolution in general; however this induces a filtration on $H^{*}\left(\Omega^{k} X\right)$ given by

$$
A_{s}=\operatorname{im}\left(H^{*}\left(\Omega^{k} X_{s}\right) \stackrel{\left(\Omega^{k} f_{s}\right)^{*}}{\rightarrow} H^{*}\left(\Omega^{k} X\right)\right) .
$$


The main theorem of [HM2] relates the associated graded of this filtration to the homology of the chain complex $\left\{\Omega^{k} P_{s}, \Omega^{k} d_{s}\right\}$ as long as $k$ is not too large. More specifically, for the fibration

there is a fundamental sequence

$$
\Omega^{k} F_{s} \rightarrow \Omega^{k} X_{s} \stackrel{\Omega^{k} p_{s-1}}{\rightarrow} \Omega^{k} X_{s-1}
$$

$$
\left.0 \rightarrow R_{s, k} \rightarrow G_{s, k} \rightarrow \Omega \operatorname{ker}\left(\Omega^{k} g_{s-1}\right)^{*}\right|_{\Omega^{k+s-1} P_{s}} \rightarrow 0 .
$$

The delicate part of [HM2] is the proof that

$$
\left.\operatorname{ker}\left(\Omega^{k} g_{s-1}\right)^{*}\right|_{\Omega^{k+s-1} P_{s}}=\operatorname{ker} \Omega^{k+s-1} d_{s-1} \quad \forall s \geq 0 .
$$

So (2.36) can be written

$$
0 \rightarrow R_{s, k} \rightarrow G_{s, k} \rightarrow \Omega \mathrm{ker} \Omega^{k+s-1} d_{s-1} \rightarrow 0 .
$$

Since $\Omega \operatorname{ker} \Omega^{k+s-1} d_{s-1} \rightarrow \Omega^{k+s} P_{s}$ is injective, it follows from (2.37) that there is a diagram in which the rows and columns are exact:

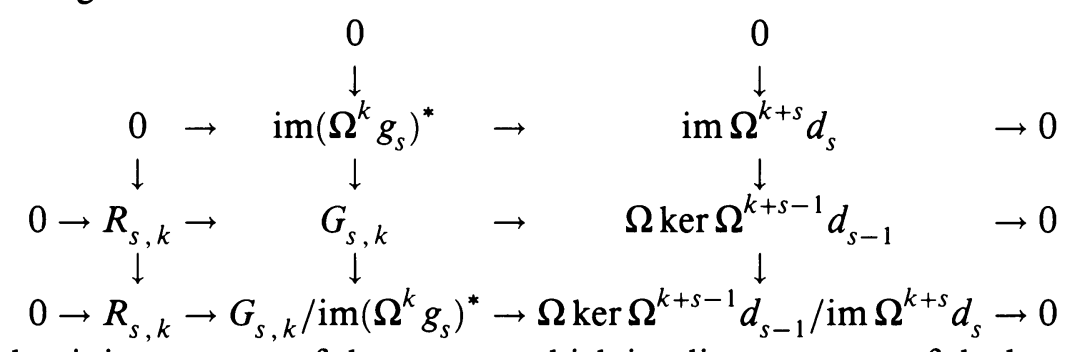

Note that it is exactness of the top row which implies exactness of the bottom row. The inclusion at the left in the bottom row implies that there is an inclusion of algebras

$$
1 \rightarrow R_{s, k} \rightarrow R_{s+1, k} \text {. }
$$

Letting $\Omega_{j}^{i}$ denote the $j$ th derived functor of the iterated algebraic loop functor $\Omega^{i}[\mathrm{Si}, \mathrm{Li}]$, the lower right-hand term is identified as $\Omega \Omega_{s}^{s+k-1} M$.

Now let $X=S^{2 n+1}, k<2 n+1$, and let $\left\{X_{s}\right\}$ be the resolution of [6A]. Since $H^{*}\left(\Omega^{k} S^{2 n+1}\right)=\lim H^{*}\left(\Omega^{k} X_{s}\right)$, Proposition 2.32(ii) follows immediately from (2.39). Using the odd primary version of Singer's sequence (see 4.1 of [Li], 2.3 of [Si]) to compute the connectivity of $\Omega \Omega_{s}^{s+k-1} M$, Proposition 2.32(i) follows.

We seek a diagram analogous to $(2.38)$ for $W(n)^{\circ}$. The following notation will be convenient: Let $P_{s}, P_{s}^{\prime}, P_{s}^{\prime \prime}$ be such that $H^{*}\left(\Omega^{3} B F_{s-1}\left(S^{2 n+1}\right)\right)=$ $U\left(P_{s}^{\prime}\right), H^{*}\left(B F_{s}\left(S^{2 n-1}\right)\right)=U\left(P_{s}^{\prime \prime}\right)$, and let $P_{s}=P_{s}^{\prime} \oplus P_{s}^{\prime \prime}$. We have $d_{s}: P_{s+1} \rightarrow$ $\Omega P_{s}$ corresponding to the composite

$$
F_{s}(W(n)) \rightarrow X_{s}(W(n)) \stackrel{g_{s}}{\rightarrow} B F_{s+1}(W(n))
$$

and by naturality, a short exact sequence of chain complexes:

$$
0 \rightarrow P_{*}^{\prime \prime} \rightarrow P_{*} \rightarrow P_{*}^{\prime} \rightarrow 0 \text {. }
$$

The proof of Proposition 2.26 hinges on the following lemma. 
Lemma 2.41. $\operatorname{ker} d_{s}=\left.\operatorname{ker} g_{s}\right|_{P_{s+1}}$.

Proof. This is equivalent to the statement that $\left.\operatorname{im} g_{s}^{*}\right|_{P_{s+1}} \rightarrow \operatorname{im} d_{s}$ is injective. This follows by a routine diagram chase in the diagram

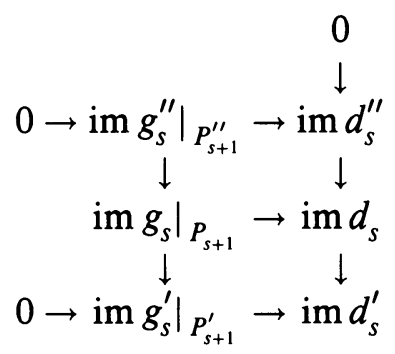

It now follows that there is a diagram

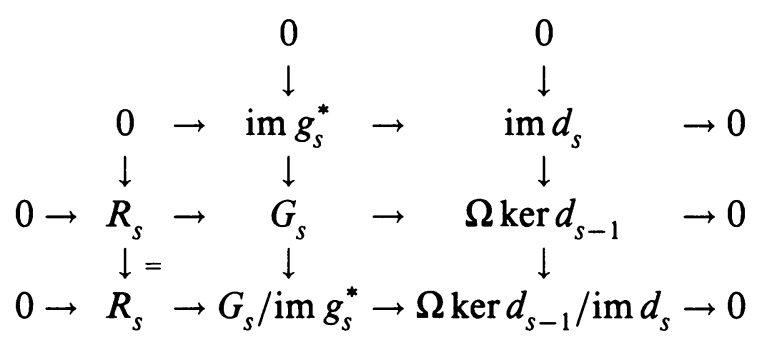

where the middle row is the fundamental sequence of the fibration

$$
F_{s}(W(n)) \rightarrow X_{s}(W(n)) \stackrel{p_{s-1}}{\rightarrow} X_{s-1}(W(n))
$$

and all the rows and columns are exact.

Now, as in Proposition 2.32(ii), the bottom left inclusion implies an inclusion $1 \rightarrow R_{s} \rightarrow R_{s+1}$, which implies Proposition 2.26(i). The connectivity of the quotient $R_{s+1} / / R_{s}$ is that of $\Omega \operatorname{ker} d_{s-1} / \operatorname{im} d_{s}$. To compute this connectivity, dualize, and consider instead the complexes of unstable injective $\mathscr{A}_{*}$-comodules corresponding to $S^{m}$, where $\mathscr{A}_{*}$ is the dual Steenrod algebra. Following Singer [Si2], an explicit construction of this complex is obtained from $\Lambda(m)$, the result being denoted by $\mathscr{A}_{*} \otimes \Sigma^{m} \Lambda(m)$. See [BK] for the odd primary case. Also in connection with this see [DM2]. Applying $\mathscr{A}_{*} \otimes()$ to diagram (2.7) shows that the connectivity of $H^{s}\left(P_{*}^{\prime} / P_{*}^{\prime \prime}\right)$ is that of $\Omega \Omega_{s}^{s+1} M$ where $M=\widetilde{H}^{*}\left(S^{2 n p-1}\right)$. Since $\Lambda(2 n+1) \oplus \Lambda(2 n-1)$ is chain equivalent to $\Lambda(2 n+1) / \Lambda(2 n-1)$, this is also the connectivity of $H^{s}\left(P_{*}\right)$ and Proposition 2.26(ii) follows.

Proof of Lemma 2.29. Let $P_{*}^{n}$ and $P_{*}^{n+1}$ be the chain complexes of free unstable $\mathscr{A}$-modules, as in (2.40), corresponding to $W(n)^{\circ}$ and $\Omega^{2 p} W(n+1)^{\circ}$ respectively. The map of resolutions $\dot{c}^{\circ}$ corresponds to a chain map

$$
P_{*}^{n+1} \rightarrow P_{*}^{n} .
$$

If $F$ is a generalized Eilenberg-Mac Lane space such that $H^{*}(F)=U(P)$, we have $\operatorname{Hom}_{\mathscr{A}}(P, \mathbf{Z} / p) \cong \pi_{*}(F)$. Thus the map of $\mathrm{E}_{2}$-terms induced by $c$ is obtained by applying $H_{*}\left(\operatorname{Hom}_{\mathscr{A}}(, \mathrm{Z} / p)\right)$ to the chain map in (2.42). We 
will show that in homological degree $s+1, P_{s+1}^{n}$ is acyclic in those dimensions in which $\operatorname{Hom}_{\mathscr{A}}\left(P_{s+1}^{n+1}, \mathbf{Z} / p\right) \neq 0$. Then Lemma 2.29 follows by the standard argument of homological algebra concerning the uniqueness up to chain homotopy of maps from a projective chain complex to an acyclic chain complex. Note that this requires a sharpening of the estimate given in the proof of Theorem 2.27. There we only showed that $P_{s+2}^{n}$ is acyclic in dimensions in which $\operatorname{Hom}_{\mathscr{A}}\left(P_{s+1}^{n+1}, \mathbf{Z} / p\right) \neq 0$.

To obtain this sharpened estimate, since $\Omega^{2 p} W(n+1)^{\cdot}$ is a minimal resolution, we need to consider $H^{s+1, *}(\Lambda(2(n+1) p+1))$. Order the monomials in $\Lambda(2(n+1) p+1)$ lexicographically as in [Ta], and let $\kappa_{i_{1}} \cdots \kappa_{i_{s+1}}$ be the leading term of a cycle in $\Lambda(2(n+1) p+1)$. We need a lemma.

Lemma 2.43. If $\kappa_{i_{1}} \cdots \kappa_{i_{s+1}}$ is the leading term of a cycle, then $\kappa_{i_{s}} \kappa_{i_{s+1}}$ has the form $\kappa_{i} \lambda_{p^{j}}$ for some $j$, and $i$ satisfies $i \geq 2 p^{j-1}$.

Assume Lemma 2.43 for the moment. Write $n+1$ uniquely as $r p^{\mu}+k$, where $1 \leq r \leq p-1$, and $k<p^{\mu}$. Since $i_{s+1}=p^{j} \leq(n+1) p^{s+1}$, we must have $p^{j} \leq p^{\mu+s+1}$. Suppose $p^{j}=p^{\mu+s+1}$. Then the dimension of $\kappa_{i_{1}} \cdots \kappa_{i_{s+1}}$ is at most $q(n+1)\left(p+p^{2}+\cdots+p^{s}\right)+q p^{\mu+s+1}+2 n p-2$. If $r \geq 2$ and $n \geq 2$, then this is less than $(2 n p-3) p^{s+1}+(p-2) p^{s}$, the dimension of the first nonzero class in $H^{s+1}\left(P_{*}^{n}\right)$.

Now consider the case where $r=1$ and suppose first that $s+1 \geq 2$. By Lemma 2.43 we have $2 p^{\mu+s} \leq i \leq(n+1) p^{s}$, which is impossible. Hence we must have $p^{j} \leq p^{\mu+s}$. Thus the dimension of $\kappa_{i_{1}} \cdots \kappa_{i_{s+1}}$ is at most

$$
q(n+1)\left(p+\cdots+p^{s}\right)+q p^{\mu+s}+2 n p-2,
$$

which is less than $(2 n p-3) p^{s+1}+(p-2) p^{s}$.

Now, if $s+1=1$, and $\kappa_{i_{1}}=\lambda_{p^{j}}$, where $p^{j}=p^{\mu+s+1}$, then the dimension of $\kappa_{i_{1}}$ is $q p^{\mu+s+1}-1+2 n p-2$. This is less than the dimension of the first nonzero class in $H^{1}\left(P_{*}^{n}\right)$ if $k \geq 2$. If $k=0,1$ then $H^{*}(W(n))$, and hence $H^{*}\left(P_{*}^{n}\right)$, is zero in this dimension. Finally, the special case $n=1$ can be handled by the method given in the proof of Theorem 2.27.

Proof of Lemma 2.43. Under the hypothesis of the lemma, $\kappa_{i_{s}} \kappa_{i_{s+1}}$ must be the leading term of a cycle in filtration $s=2$ by 2.3, 2.4 of [HM1]. The homology of $\Lambda$ is the stable $E_{2}$-term of the Adams spectral sequence for the sphere, and in filtration $s=2$ this was first computed in [L]. It consists of products $h_{i} h_{j}$, $i \leq j-2, a_{0} h_{j}, j>0$, elements $a_{0} a_{0}$ and $a_{1}$, and Massey products involving the elements $\left\{h_{i}\right\}$. The element $\left\{h_{i}\right\}$ has bidegree $\left(1, q p^{i}\right)$ and is represented in $\Lambda$ by $\lambda_{p^{i}}$. Each of the above elements can be easily represented by a cycle in $\Lambda^{2, *}$ in admissible form, and the desired conclusion is obtained by inspecting the leading terms. The only cycles in $\Lambda^{2, *}(m)$ which are 0 in $\Lambda$ are $d(\kappa)$ for 
some $\kappa=\lambda_{j}$ or $\mu_{j}$ and the leading terms of these satisfy the conclusion as well.

\section{3. $J$-HOMOLOGY OF STUNTED LENS SPACES}

In this section we recall the basic properties of the spectrum $J$, the connection between $J$-homology and $v_{1}$-periodic homotopy, and the calculation of the $J$-homology of stunted lens spaces. Corollary 1.2 will follow immediately from this.

Let $\ell$ denote a wedge summand of the spectrum representing complex connective $K$-theory, and consider the Adams operation

$$
\psi^{k}: \ell \rightarrow \ell, \quad k \in \mathbf{Z},
$$

where $k$ is a $(p-1)$ st root of unity $\bmod (p)$ but not $\bmod \left(p^{2}\right)$. Then we get $\left(\psi^{k}-1\right): \ell \rightarrow \ell$. This lifts to a map $\left(\psi^{k}-1\right): \ell \rightarrow \Sigma^{q} \ell$ and $J$ is defined as the fiber of this lifting. $J$ is a connective spectrum with the following homotopy groups, where $\nu(i)$ is defined by $i=p^{\nu(i)} s$, where $p$ does not divide $s$ :

$$
\begin{aligned}
\pi_{0} J & =\mathbf{Z}_{(p)}, \\
\pi_{q i-1} J & =\mathbf{Z} / p^{\nu(i)+1}, \quad i>0, \\
\pi_{n} J & =0 \text { if } n \neq 0 \text { and } n \neq 1 \quad(\bmod q) .
\end{aligned}
$$

The image of the classical $J$ homomorphism is mapped isomorphically onto these groups by the map $S^{0} \rightarrow J$. See [D] or [R1] for more details concerning the properties of $J$.

By combining results from [DMM], [R3], and [B] one can prove the following stable result.

Proposition 3.2. Let $X$ be a p-local spectrum. Then there is an isomorphism

$$
v_{1}^{-1} \pi_{*}^{S}(X ; \mathbf{Z} / p) \cong v_{1}^{-1} J_{*}(X ; \mathbf{Z} / p) \text {. }
$$

A version of this is true for $p=2$ as well and this generalizes Theorem 6.2 of [M1] to an arbitrary spectrum $X$. Since the $J$-homology of a spectrum $X$ can be calculated from the $\ell$-homology, this gives a convenient way of computing the stable $v_{1}$-periodic homotopy groups of $X$.

Now we compute $J_{*}\left(\left(B \Sigma_{p}\right)_{q m-1}^{q n}\right)$ where $\left(B \Sigma_{p}\right)_{q m-1}^{q n}$ denotes the $q n$ skeleton of $B \Sigma_{p}$ localized at $p$ with the $q(m-1)$ skeleton pinched to a point. Thus $\left(B \Sigma_{p}\right)_{q m-1}^{q_{n}}$ is a space with top cell in dimension $q n$, and bottom cell in dimension $q m-1$, for $1 \leq m \leq n \leq \infty$. To simplify notation we will denote this complex by $B_{q m-1}^{q n}$, or just $B$ if $n=\infty$ and $m=1$.

To begin, using the methods of $[\mathrm{K}]$ for example, one easily calculates the $\ell$-homology of $B$ to obtain

$$
\ell_{q i-1}(B)=\mathrm{Z} / p^{i}, \quad \ell_{n}(B)=0 \quad \text { if } n \not \equiv 1 \quad(\bmod q) .
$$

In $[R 1]$ it is shown that the fibration

$$
\ell \wedge B \rightarrow \Sigma^{q} \ell \wedge B \rightarrow \Sigma J \wedge B
$$


induces the following long exact sequence of homotopy groups:

$$
0 \rightarrow J_{q i-1}(B) \rightarrow \ell_{q i-1}(B) \stackrel{\times i}{\rightarrow} \ell_{q(i-1)-1}(B) \rightarrow J_{q i-2}(B) \rightarrow 0
$$

or

$$
0 \rightarrow J_{q i-1}(B) \rightarrow \mathbf{Z} / p^{i} \stackrel{\times i}{\rightarrow} \mathbf{Z} / p^{i-1} \rightarrow J_{q i-2}(B) \rightarrow 0 .
$$

It follows that

$$
\begin{aligned}
J_{q i-1}(B) & =\mathbf{Z} / p^{\nu(i)+1}, \quad J_{q i-2}(B)=\mathbf{Z} / p^{\nu(i)}, \\
J_{n}(B) & =0 \quad \text { if } n \not \equiv-1,-2 \bmod q .
\end{aligned}
$$

To compute $J_{*}\left(B_{q m-1}^{q n}\right)$ first look at the cofiber sequence

$$
B^{q(m-1)} \rightarrow B \rightarrow B_{q m-1} .
$$

The long exact sequence in $\ell$-homology gives

$$
\begin{aligned}
\ell_{q i-1}\left(B_{q m-1}\right) & =\mathbf{Z} / p^{i-m+1}, \quad i \geq m, \\
\ell_{q i-1}\left(B^{q m}\right) & =\mathbf{Z} / p^{i}, \quad i \leq m, \\
\ell_{q i-1}\left(B^{q m}\right) & =\mathbf{Z} / p^{m}, \quad i \geq m .
\end{aligned}
$$

Similarly, we get

$$
\ell_{q i-1}\left(B_{q m-1}^{q n}\right)=\mathbf{Z} / p^{n-m+1}, \quad i \geq n .
$$

So the long exact sequence in homotopy groups applied to the fiber sequence

$$
J \wedge B_{q n-1} \rightarrow \ell \wedge B_{q n-1} \rightarrow \Sigma^{q} \ell \wedge B_{q n-1}
$$

looks like

$$
0 \rightarrow J_{q i-1}\left(B_{q n-1}\right) \rightarrow \mathbf{Z} / p^{i-n+1} \stackrel{\times p^{(i)}}{\rightarrow} \mathbf{Z} / p^{i-n} \rightarrow J_{q i-2}\left(B_{q n-1}\right) \rightarrow 0,
$$

which yields

$$
\begin{aligned}
J_{q i-1}\left(B_{q n-1}\right) & =\mathbf{Z} / p^{\min (\nu(i)+1, i-n+1)}, \\
J_{q i-2}\left(B_{q n-1}\right) & =\mathbf{Z} / p^{\min (\nu(i), i-n)}, \\
J_{k}\left(B_{q n-1}\right) & =0 \text { if } k \not \equiv-1,-2 \bmod q .
\end{aligned}
$$

Also the long exact sequence in homotopy groups for the fiber sequence

$$
J \wedge B_{q m-1}^{q n} \rightarrow \ell \wedge B_{q m-1}^{q n} \rightarrow \Sigma^{q} \ell \wedge B_{q m-1}^{q n}
$$

yields for $i>n$

$$
\begin{aligned}
J_{q i-1}\left(B_{q m-1}^{q n}\right) & =\mathbf{Z} / p^{\min (\nu(i)+1, n-m+1)}, \\
J_{q i-2}\left(B_{q m-1}^{q n}\right) & =\mathbf{Z} / p^{\min (\nu(i)+1, n-m+1)}, \\
J_{k}\left(B_{q m-1}^{q n}\right) & =0 \text { if } k \not \equiv-1,-2 \bmod q .
\end{aligned}
$$


To compute $v_{1}^{-1} J_{*}\left(B^{q n} ; \mathbf{Z} / p\right)$, first recall that for any spectrum $X$ we have defined $\pi_{n}(X ; \mathbf{Z} / p)=\left[M^{n}, X\right]$ and there is a universal coefficient sequence

$$
0 \rightarrow \pi_{n}(X) \otimes \mathbf{Z} / p \rightarrow \pi_{n}(X, \mathbf{Z} / p) \rightarrow \operatorname{Tor}\left(\pi_{n-1}(X), \mathbf{Z} / p\right) \rightarrow 0 .
$$

It follows immediately that

$$
\begin{aligned}
J_{q i-2}\left(B^{q n} ; \mathbf{Z} / p\right) & =\mathbf{Z} / p, \\
J_{q i-1}\left(B^{q n} ; \mathbf{Z} / p\right) & =\mathbf{Z} / p \oplus \mathbf{Z} / p, \\
J_{q i}\left(B^{q n} ; \mathbf{Z} / p\right) & =\mathbf{Z} / p .
\end{aligned}
$$

To invert $v_{1}$, let $M$ denote the mod $p$ Moore spectrum with bottom cell in dimension zero, and observe that by Spanier-Whitehead duality $\left[M^{n}, X\right] \cong$ $\left[S^{n}, X \wedge M\right]$, and $v_{1}$ is self dual. The Adams maps $v_{1}: M \rightarrow \Sigma^{-q} M$ acts on $\ell_{k}(X \wedge M)=\pi_{k}(\ell \wedge X \wedge M)$ by taking a map $S^{k} \rightarrow \ell \wedge X \wedge M$ and

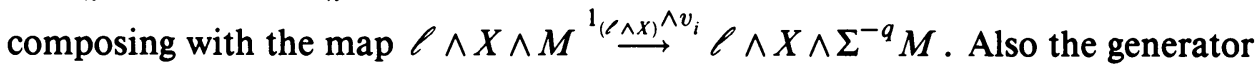
of $\pi_{q}(\ell)$, which we have denoted by $v_{1}$, acts on $\pi_{k}(\ell \wedge X \wedge M)$ by taking a map $S^{k} \rightarrow \ell \wedge X \wedge M$, smashing it with $S^{q} \rightarrow \ell$ to get a map $S^{k+q} \rightarrow \ell \wedge \ell \wedge X \wedge M$, then composing with the ring spectrum multiplication map $\ell \wedge \ell \rightarrow \ell$ smashed with $X \wedge M$. In [DM] it is proved that these two actions coincide. The latter action of $v_{1}$ on $\ell_{*}(X)$ coincides on the Adams $\mathrm{E}_{2}$-level to the action of an element $b \in \operatorname{Ext}_{E\left(Q_{0}, Q_{1}\right)}^{1, q+1}(\mathbf{Z} / p, \mathbf{Z} / p)$ on $\operatorname{Ext}_{E\left(Q_{0}, Q_{1}\right)}^{s, t}\left(H^{*} X, \mathbf{Z} / p\right)$.

So, in $J_{*}\left(B^{q n} ; \mathbf{Z} / p\right)$, the $v_{1}$ action on the elements which map nontrivially into $\ell_{*}\left(B^{q n} ; \mathbf{Z} / p\right)$ can be read off in $\operatorname{Ext}_{E\left(Q_{0}, Q_{1}\right)}^{s, t}\left(H^{*}\left(B^{q n}\right) \otimes E\left(Q_{0}\right), \mathbf{Z} / p\right)$ and the $v_{1}$ action of the elements in $J_{*}\left(B^{q n} ; \mathbf{Z} / p\right)$ which are in the image of $\ell_{*-(q-1)}\left(B^{q n} ; \mathbf{Z} / p\right)$ can be read off from $\operatorname{Ext}_{E\left(Q_{0}, Q_{1}\right)}^{s, t-(q-1)}\left(H^{*}\left(B^{q n}\right) \otimes E\left(Q_{0}\right), \mathbf{Z} / p\right)$. The result is

Proposition 3.9. $v_{1}^{-1} J_{*}\left(\left(B \Sigma_{p}\right)^{q n} ; \mathbf{Z} / p\right)$ is isomorphic to a free module over $\mathbf{Z} / p\left[v_{1}, v_{1}^{-1}\right]$ on four generators in dimensions $q n-2, q n-1, q n-1, q n$.

Combining this with Proposition 3.2 and Theorem 1.1 we get Corollary 1.2.

\section{ThE PROOF OF THEOREM 1.3}

In order to prove Theorem 1.3 we will need two results. The first result is known as the odd primary vector field theorem.

Proposition 4.1. (a) There exists a map

$$
a_{k}: S^{q k-1} \rightarrow B_{q(k-\nu(k)-1)}
$$

with the following properties:

(i) $a_{k}$ is nontrivial in $\bmod p$ homology, and

(ii) $S^{q k-1} \stackrel{a_{k}}{\rightarrow} B_{q(k-\nu(k)-1)} \rightarrow B_{q(k-\nu(k)-1)} \wedge \ell$ represents a generator of $\ell_{q k-1}\left(B_{q(k-\nu(k)-1)}\right)$. 
(b) There does not exist a map $S^{q k-1} \rightarrow B_{q(k-\nu(k)-1)-1}$ nontrivial in mod $p$ homology.

A proof of part (i) can be found in, for example, [D], and is based on the fact that the Thom complex of the canonical complex line bundle over a skeleton of $B \Sigma_{p}$ is a stunted $B_{m}^{n}$. The second part of (a) follows immediately from part (i). Part (b) is originally due to Toda. An elegant proof can be made using the Adams operations $\psi^{k}$ in $\ell$-theory, based on the proof of the $p=2$ version given in $[\mathrm{MM}]$. We will only make use of part (a).

The second result concerns the properties of the map $P: B \Sigma_{p} \rightarrow B \Sigma_{p}$ which is the identity on $B \Sigma_{p}$ smashed with the degree $p$ map on the sphere. If we view $B \Sigma_{p}$ as a space rather than a spectrum, then $P$ can be defined after just one suspension, and the following proposition holds true after two suspensions. Let $c: B_{n q-1} \rightarrow B_{(n+1) q-1}$ denote collapse, $i: B^{q n} \rightarrow B^{q(n+1)}$ denote inclusion.

Proposition 4.2. (a) For $n \geq 1$, there exists a map $\phi_{n}: B_{(n+1) q-1} \rightarrow B_{n q-1}$ with the following properties:

(i) $\phi_{n}$ extends multiplication by $p$, i.e.,

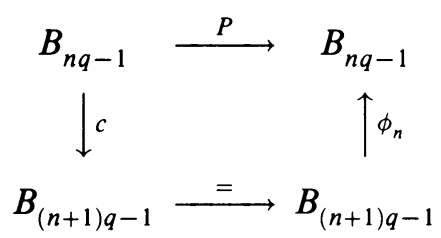

commutes.

(ii) $\phi_{n}$ has positive Adams filtration; i.e., $\phi_{n}$ is 0 in $\bmod p$ homology.

(iii) $\phi_{n}$ induces an isomorphism in $K$-theory and multiplication by $p$ in $\ell$-theory.

(b) For $1 \leq m \leq n$, there exists a map

$$
\phi_{m}^{n}: B_{q(m+1)-1}^{q(n+1)} \rightarrow B_{q m-1}^{q n}
$$

with the following properties:

(i) The following diagram commutes:

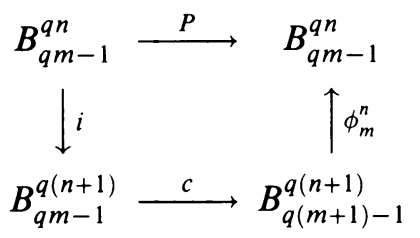

(ii) $\phi_{m}^{n}$ has positive Adams filtration.

(iii) $\phi_{m}^{n}$ induces an isomorphism in $K$-theory.

(iv) $\phi_{m}^{n}$ induces an isomorphism in $\ell$-theory in dimensions $\geq q(n+1)-1$ and multiplication by $p$ in dimensions $<q(n+1)-1$.

Proof of Proposition 4.2. $\phi_{n}$ is just the usual extension over the cofiber which exists because the composite $\Sigma M_{n q-1}^{n q} \stackrel{i}{\rightarrow} \Sigma B_{n q-1} \stackrel{P}{\rightarrow} \Sigma B_{n q-1}$ is null homotopic. 
Properties (ii) and (iii) are readily checked by applying $H^{*}(), \ell_{*}()$, and $K_{*}()$ to diagram (4.3).

Since the composite $\Sigma B_{q m-1}^{q(n+1)} \stackrel{P}{\rightarrow} \Sigma B_{q m-1}^{q(n+1)} \stackrel{c}{\rightarrow} \Sigma M^{q(n+1)}$ is null, there exists a colifting $\Sigma^{2} B_{q m-1}^{q(n+1)} \stackrel{f}{\rightarrow} \Sigma^{2} B_{q m-1}^{q n}$. The composite

$$
\Sigma^{2} M^{q m} \stackrel{i}{\rightarrow} \Sigma^{2} B_{q m-1}^{q(n+1)} \stackrel{f}{\rightarrow} \Sigma^{2} B_{q m-1}^{q n} \stackrel{i}{\rightarrow} \Sigma^{2} B_{q m-1}^{q(n+1)}
$$

is null since $i \circ f=p$. But $i: \Sigma^{2} B_{q m-1}^{q n} \rightarrow \Sigma^{2} B_{q m-1}^{q(n+1)}$ is $q(n+1)-1$ connected so

$$
f \circ i: \Sigma^{2} M^{q m} \rightarrow \Sigma^{2} B_{q m-1}^{q(n+1)} \stackrel{f}{\rightarrow} \Sigma^{2} B_{q m-1}^{q n}
$$

is null. Thus there exists an extension over the cofiber $\Sigma^{2} B_{q(m+1)-1}^{q(n+1)} \rightarrow \Sigma^{2} B_{q m-1}^{q n}$ and this is our map $\phi_{m}^{n}$. Again properties (b)(ii)-(iv) are readily checked.

We now prove a stable version of Theorem 1.3. For any space or spectrum $X$ there is a Hurewicz homomorphism induced by $S^{0} \rightarrow J$.

Proposition 4.5. The Hurewicz homomorphism

$$
\pi_{q k-1}^{S}\left(B_{n q-1}\right) \rightarrow J_{q k-1}\left(B_{n q-1}\right)
$$

is surjective for all $n \geq 1, k \geq 1$.

Proof. Consider the composite map of spectra

$$
B_{n q-1} \rightarrow B_{n q-1} \wedge J \rightarrow B_{n q-1} \wedge \ell .
$$

First assume $\nu(k)+1 \geq k-n+1$. By (3.5) and (3.7) the second map induces an isomorphism in homotopy, so it suffices to find an element in $\pi_{q k-1}\left(B_{n q-1}\right)$ which maps to a generator of $\ell_{q k-1}\left(B_{n q-1}\right)$. The vector field theorem says there is a map $a_{k}: S^{q k-1} \rightarrow B_{q(k-\nu(k)-1)}$.

Since $n>k-\nu(k)-1$ we can compose $a_{k}$ with the collapse map $B_{q(k-\nu(k)-1)}$ $\stackrel{c}{\rightarrow} B_{n q-1}$ to get a map with the desired properties.

Now assume $\nu(k)+1<k-n+1$. In this case (3.5) and (3.7) show that the second map induces multiplication by $p^{k-n-\nu(k)}$ from $\mathbf{Z} / p^{\nu(k)+1}$ to $\mathbf{Z} / p^{k-n+1}$. So we need an element of $\pi_{q k-1}\left(B_{n q-1}\right)$ which maps to $p^{k-n-\nu(k)}$ times the generator of $\ell_{q k-1}\left(B_{n q-1}\right)$. We use the composite

$$
\phi_{n} \circ \phi_{n+1} \circ \cdots \circ \phi_{k-\nu(k)-2}: B_{q(k-\nu(k))-1} \rightarrow B_{q n-1} .
$$

Again the vector field theorem gives $a_{k}: S^{q k-1} \rightarrow B_{q(k-\nu(k))-1}$ and the composite

$$
\phi_{n} \circ \cdots \circ \phi_{k-\nu(k)-2} \circ a_{k}: S^{q k-1} \rightarrow B_{q n-1}
$$

has the right properties since $\phi_{i}$ induces multiplication by $p$ in $\ell_{*}$-theory.

From this we get another stable result. 
Proposition 4.6. The Hurewicz map $\pi_{q k-1}^{S}\left(B_{(j+1) q-1}^{(n+j) q}\right) \rightarrow J_{q k-1}\left(B_{(j+1) q-1}^{(n+j) q}\right)$ is surjective for all $k \geq 1, n \geq 1$ and $j \geq 0$. If $n \geq 1, j \geq 0$ and $k \geq n+j+1+\nu(k)$, then

$$
\pi_{q k-2}^{S}\left(B_{(j+1) q-1}^{(n+j) q}\right) \rightarrow J_{q k-2}\left(B_{(j+1) q-1}^{(n+j) q}\right)
$$

is surjective.

Before giving the proof we state a lemma.

Lemma 4.7. $\pi_{q k-1}^{S}\left(B_{n q-1}\right)$ has no elements of Adams filtration greater than $k-n$.

Proof of Lemma 4.7. This follows immediately from Theorem 1 of [L] since $H^{*}\left(B_{n q-1}\right)$ is $E\left(Q_{0}\right)$ free.

Proof of Proposition 4.6. Consider the cofiber sequence of spectra

$$
B_{(j+1) q-1}^{(n+j) q} \rightarrow B_{(j+1) q-1} \rightarrow B_{(n+j+1) q-1} .
$$

By Proposition 4.5 there is an element $\alpha \in \pi_{q k-1}^{S}\left(B_{(j+1) q-1}\right)$ of Adams filtration $\max (0, k-j-\nu(k)-1)$ which maps to a generator of $J_{q k-1}\left(B_{(j+1) q-1}\right)$. If $\nu(k)<n$ then $\alpha$ maps to $\pi_{q k-1}^{S}\left(B_{(n+j+1) q-1}\right)$ above the vanishing line of Lemma 4.7; hence $\alpha$ must lift to an element in $\pi_{q k-1}^{S}\left(B_{(j+1) q-1}^{(n+j) q}\right)$ as required. If $\nu(k) \geq n$, then $p^{b} \cdot \alpha$, where $b=(k-n-j)$-Adams filtration $(\alpha)$, maps to an element above the vanishing line, hence lifts to yield the desired element.

For the second assertion of Proposition 4.6, consider the boundary map in the long exact sequence obtained by applying $J_{*}$ to (4.8). If $k \geq n+j+\nu(k)+1$, then this boundary map

$$
J_{q k-1}\left(B_{(n+j+1) q-1}\right) \stackrel{\partial}{\rightarrow} J_{q k-2}\left(B_{(j+1) q-1}^{(n+j) q}\right)
$$

is surjective, and since

$$
\pi_{q k-1}^{S}\left(B_{(n+j+1) q-1}\right) \rightarrow J_{q k-1}\left(B_{(n+j+1) q-1}\right)
$$

is surjective as well by Proposition 4.5, we have that

$$
\pi_{q k-2}^{S}\left(B_{(j+1) q-1}^{(n+j) q}\right) \rightarrow J_{q k-2}\left(B_{(j+1) q-1}^{(n+j) q}\right)
$$

is surjective.

We are now ready to finish the proof of Theorem 1.3.

Proof of Theorem 1.3. Consider the composite map of spaces $B^{q n} \rightarrow \Omega^{2 n+1} S^{2 n+1}$ $\rightarrow Q\left(B^{q n}\right)$ where the map on the left is the Dyer-Lashof map and the map on the right is the Hopf-James map. The composite is just the natural inclusion. The map on the left extends to a map

$$
\Omega^{2 n+1} \Sigma^{2 n+1} B^{q n} \stackrel{\mu}{\rightarrow} \Omega^{2 n+1} S^{2 n+1} \rightarrow Q\left(B^{q n}\right) .
$$


Fix $q k-1$. By the Freudenthal suspension theorem for the prime $p$ any map $S^{q k-1} \rightarrow Q\left(B_{(j+1) q-1}^{(j+n) q}\right)$ factors through $\Omega^{2 n+1} \Sigma^{2 n+1}\left(B_{(j+1) q-1}^{(j+n) q}\right)$ if

$$
2 n+q k<p[2 n+(j+1) q]-1 .
$$

Furthermore, the stable map

$$
Q\left(\phi_{1}^{n} \circ \cdots \circ \phi_{j}^{j+n-1}\right): Q B_{(j+1) q}^{(j+n) q} \rightarrow Q B^{q n}
$$

induces an isomorphism in $J_{q k-1}()$ if

$$
q k-1>(j+n) q \text {. }
$$

Given the integer $k$, we must find a $j$ which simultaneously satisfies (4.9) and (4.10). This amounts to finding an integer $j$ such that $(k-n) / p-1<j<$ $k-n$ which we can always do. Thus we apply Proposition 4.6 to get a map

$$
S^{q k-1} \rightarrow \Omega^{2 n+1} \Sigma^{2 n+1} B_{(j+1) q-1}^{(j+n) q},
$$

which we then compose with $\mu \circ \Omega^{2 n+1} \Sigma^{2 n+1}\left(\phi_{j}^{j+n-1} \circ \cdots \circ \phi_{1}^{n}\right)$ to get an element in $\pi_{q k-1}\left(\Omega^{2 n+1} S^{2 n+1}\right)$ which maps to a generator of $\dot{J}_{q k-1}\left(B^{q n}\right)$, proving the first part of Theorem 1.3.

To prove the second part consider the composite

$$
\Omega^{2 n+1} \Sigma^{2 n+1} B_{(j+1) q-1}^{q(n+j)} \rightarrow \Omega^{2 n+1} \Sigma^{2 n+1} B^{q n} \rightarrow \Omega^{2 n+1} S^{2 n+1} \rightarrow Q B^{q n} .
$$

By Proposition 4.6, there is an element $\alpha: S^{q k-2} \rightarrow Q\left(B_{(j+1) q-1}^{(n+j) q}\right)$ whose Hurewicz image is a generator if

$$
k \geq n+j+1+\nu(k) .
$$

Consider the diagram

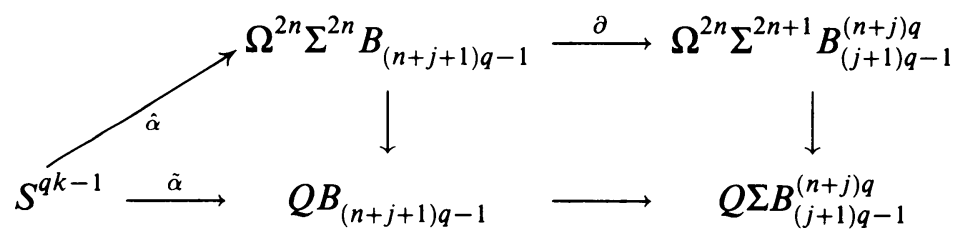

and recall from the proof of Proposition 4.6 that $\alpha$ is obtained as the composite of the bottom row for some map $\tilde{\alpha}$. If $\tilde{\alpha}$ has a lifting $\hat{\alpha}$ then the adjoint of $\partial \circ \hat{\alpha}$ would yield an element in $\pi_{q k-2}\left(\Omega^{2 n+1} S^{2 n+1}\right)$ which would then map to the generator of $J_{q k-2}\left(B^{q n}\right)$. But $\tilde{\alpha}$ will be in the range to have such a lifting if

$$
q k-1+2 n<p[2 n+(n+j+1) q-1]-1
$$

or equivalently,

$$
q k<\left(2 p^{2}-2\right) n+p q(j+1)-p .
$$

After a little arithmetic, one sees that given $k$ such that $k \geq 1+n+\nu(k)$ as in the hypothesis, it is possible to find a $j$ simultaneously satisfying (4.11) and (4.13) which completes the proof of the second part of Theorem 1.3. 


\section{BIBLIOGRAPHY}

[A] J. F. Adams, On the groups $J(X)$. IV, Topology 5 (1966), 21-71.

[B] A. K. Bousfield, The localization of spectra with respect to homology, Topology 18 (1979), 257-281.

[Ba] W. D. Barcus, On a theorem of Massey and Peterson, Quart. J. Math. 19 (1968), 33-41.

[Be] M. Bendersky, Unstable towers in the odd primary homotopy groups of spheres, Trans. Amer. Math. Soc. 287 (1985), 529-542.

[BK] A. K. Bousfield and D. M. Kan, The homotopy spectral sequence of a space with coefficients in a ring, Topology 11 (1972), 79-106.

[6A] A. K. Bousfield, E. B. Curtis, D. M. Kan, D. G. Quillen, D. C. Rector, and J. W. Schlesinger, The $\bmod p$ lower central series and the Adams spectral sequence, Topology 5 (1966), 331342.

[C] Fred Cohen, The unstable decomposition of $\Omega^{2} \Sigma^{2} X$ and its applications, Math. Z. 182 (1983), 553-568.

[CN] F. Cohen and J. Neisendorfer, A note on desuspending the Adams map, Math. Proc. Cambridge Philos. Soc. 99 (1986), 59-64.

[D] D. Davis, Odd primary bo-resolutions and $K$-theory localizations, Illinois J. Math. 30 (1986), 79-100.

[DM] D. Davis and M. Mahowald, $v_{1}$ and $v_{2}$ periodicity in stable homotopy theory, Amer. J. Math. 103 (1981), 615-659.

[DM2] _ , $v_{1}$-periodicity in the unstable Adams spectral sequence (to appear).

[DMM] D. Davis, M. Mahowald, and H. Miller, Mapping telescopes and K-theory localization, Ann. of Math. Stud., no. 113, Princeton Univ. Press, Princeton, N.J., 1987, pp. 152-167.

[G1] B. Gray, Unstable families related to the image of $J$, Math. Proc. Cambridge Philos. Soc. 96 (1984), 95-113.

[G2] _ On the sphere of origin of infinite families in the homotopy groups of spheres, Topology 8 (1969), 219-232.

[H] J. R. Harper, H-spaces with torsion, Mem. Amer. Math. Soc. No. 233 (1979).

[HM1] J. R. Harper and H. R. Miller, On the double suspension homomorphism at odd primes, Trans. Amer. Math. Soc. 273 (1982), 319-331.

[HM2] __ Looping spaces with cohomology $U(M)$ (to appear).

[K] R. Kane, Operations in connective K-theory, Mem. Amer. Math. Soc. No. 254 (1981).

[Ku] N. J. Kuhn, The geometry of James-Hopf maps, Pacific J. Math. 102 (1982), 397-412.

[L] A. Liulevicius, Zeroes of the cohomology of the Steenrod algebra, Proc. Amer. Math. Soc. 14 (1963), 972-976.

[L2] - The factorization of cyclic reduced powers by secondary cohomology operations, Mem. Amer. Math. Soc. No. 42 (1962).

[Li] Hu Hsiung Li, The construction of a certain homology of the Steenrod algebra, Soochow J. Math. 9 (1983), 159-165.

[M1] Mark Mahowald, The image of $J$ on the EHP sequence, Ann. of Math. (2) 116 (1982), 65-112.

[M2] _ 178.

[Mi1] H. R. Miller, On relations between Adams spectral sequences, with an application to the stable homotopy of a Moore space, J. Pure Appl. Algebra 20 (1981), 287-312.

[Mi2] _ A localization theorem in homological algebra, Math. Proc. Cambridge Philos. Soc. 84 (1978), 73-84.

[MM] M. Mahowald and R. Milgram, Operations which detect $S_{q}^{4}$ in connective K-theory, Quart. J. Math. Oxford Ser. (2) 27 (1976), 415-432. 
[MP1] W. S. Massey and F. P. Peterson, The cohomology structure of certain fibre spaces. I, Topology 4 (1965), 47-65.

[MP2] _ The mod 2 cohomology structure of certain fibre spaces, Mem. Amer. Math. Soc. No. 74 (1967).

[MT] M. Mahowald and R. Thompson, A commentary on 'The image of $J$ in the EHP sequence', Seattle Proceedings, 1985, Lecture Notes in Math., vol. 1286, Springer-Verlag, Berlin and New York.

[R1] D. Ravenel, Complex cobordism and the stable homotopy groups of spheres, Academic Press, New York, 1986.

[R2] _ Streamlining the EHP sequence by excluding $v_{1}$-periodicity (to appear).

[R3] _ Localization with respect to certain periodic homology theories, Amer. J. Math. 106 (1984), 351-414.

[Se] P. S. Selick, Odd primary torsion in $\pi_{k} S^{3}$, Topology 17 (1978), 407-412.

[Si] W. M. Singer, Iterated loop functors and the homology of the Steenrod algebra, J. Pure Appl. Algebra 11 (1977), 83-101.

[Si2] _ The algebraic EHP sequence, Trans. Amer. Math. Soc. 201 (1975), 367-382.

[T] H. Toda, On the double suspension $E^{2}$, J. Inst. Polytech. Osaka City Univ. 7 (1956), 103145.

[Ta] M. C. Tangora, Computing the homology of the lambda algebra, Mem. Amer. Math. Soc. No. 337 (1985).

[W] G. Whitehead, On the homotopy groups of spheres and rotation groups, Ann. of Math. (2) 43 (1942), 634-640.

Department of Mathematics, Northwestern University, Evanston, Illinois 60208 